\title{
A simplified model of a fueldraulic actuation system with application to load estimation
}

\author{
Tomas Puller ${ }^{1}$ and Andrea Lecchini-Visintini ${ }^{2}$
}

\begin{abstract}
In this work, a simplified model of the compressor variable stator vane fueldraulic actuation system of a jet engine is presented. The actuation system is a sub-assembly of the engine's Hydro-Mechanical Unit (HMU). A unique characteristic of the actuator is an internal cooling flow which prevents the overheating of fuel. It is shown that the effect of the cooling flow is well represented by a static input nonlinearity. The resulting model is of the Hammerstein structure. It is then shown that the model can be used for the estimation of the actuator's external load. The results are validated using an accurate real system simulator.
\end{abstract}

\section{Keywords}

Hydraulic actuators, Fueldraulic systems, Position control, Hammerstein model, Aerospace simulation

\section{Introduction}

Hydraulic servo-systems are characterised by high power-to-weight ratios, and the ability to generate forces in locations which are remote and difficult to reach. For these reasons hydraulic servo-systems are widely used in aviation for the actuation of control surfaces and other moving parts. An introduction to hydraulic systems, hydraulic components, physical models, and linearisation techniques, can be found in Merritt ${ }^{1}$ and Jalali and Kroll ${ }^{2}$. Modelling and control applications for hydraulic systems have been reported under different approaches. These include: control-oriented models, ${ }^{3-6}$ and PID, ${ }^{7-9}$ robust, $^{10-17}$ adaptive and nonlinear ${ }^{18-26}$ control designs. In some of these contributions, full position and pressure feedback is adopted. However, in aerospace applications, requiring low complexity and high reliability, the use of pressure sensors within the actuators is often avoided.

In this work, the modelling aspects of the Variable Stator Vanes (VSV) fueldraulic actuation system of a Rolls-Royce Trent 1000 jet engine are developed. Here, the term fueldraulic indicates that, in order to reduce overall weight, the actuator uses pressurised fuel in place of an hydraulic fluid. The purpose of the VSV actuator is to position the jet engine stator vanes in such a way that they attain an optimal angle of attack in all engine operating conditions. The vanes are mounted in a casing, so that rotation along their shroud-tip axis is enabled, and are joined in a unison ring which enables their uniform actuation. The actuator is located on the intermediate pressure compressor case, which, during engine operation, is a relatively high temperature zone. As a consequence, a unique feature of this actuator is that its piston has an orifice whose purpose is to establish a cooling flow inside the actuator in order to prevent fuel inside the actuator from overheating. The cooling flow has a substantial effect on the behaviour of the actuator, similar to that of a sizeable internal leakage. The actuator is also characterised by asymmetric profiles of its servo valve's orifices, resulting in separate low- and high-flow zones, which induce a safe slow retraction of the actuator in the case of electric power loss.

The internal leakage is a feature in many hydraulic actuators' models. In the context of QFT and LPV robust control design, ${ }^{10-13,17}$ previous contributions have taken into account the effect of the internal leakage on the linearised dynamics of the hydraulic actuator for the design of the local controllers. In more complex adaptive and nonlinear design methods based on the use of global nonlinear models ${ }^{18-22,24,25}$ the internal leak is simply included in the full set of nonlinear equations of the model. In contrast with previous works, in this contribution, a simplified model of the global dynamics of the actuation system is developed, in which the effect of the cooling flow is well represented by a static input nonlinearity. The resulting model is of the Hammerstein structure, see e.g. Giri and $\mathrm{Bai}^{27}$. The input nonlinearity turns out to be either a 'deadzone' or a 'preload' nonlinearity depending on the actuator's external load. To the extent of the authors knowledge, such representation of an internal flow or leakage on the global inputoutput dynamics of the actuator is a new insight into the modelling of hydraulic actuators. In the literature concerned with the modelling for control of hydraulic actuators, deadzone nonlinearities have been considered before, but only to take into account

\footnotetext{
$1,{ }^{2}$ University of Leicester, UK

Corresponding author:

Andrea Lecchini-Visintini Department of Engineering, University of Leicester, University Road, Leicester, LE1 7RH, UK.

Email: alv1@leicester.ac.uk
} 


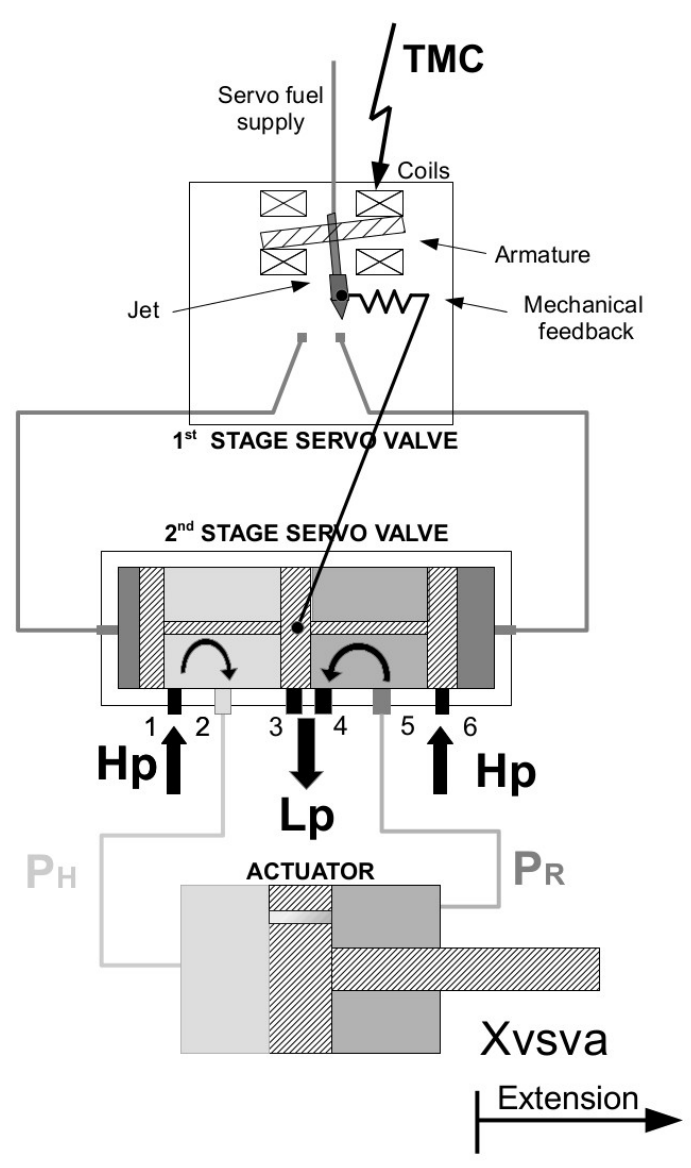

Figure 1. The actuation system: two-stage servo valve and VSV actuator depicted in extending mode.

manufacturing imperfection such as valve overlaps (see e.g. Liu and Daley ${ }^{7}$, Lee et al. ${ }^{8}$, Ye et al. ${ }^{9}$, Mohanty and $\mathrm{Yao}^{20}$ ). It will be shown that the simplified model can be used to estimate the actuator's external load. This task turns out to be a special case of identification of a Hammerstein system with an input nonlinearity of known structure ${ }^{28}$. In $\mathrm{Bai}^{28}$ the identification method is developed for a one-dimensional parametrisation encompassing different types of input nonlinearities. Here, the algorithms are tailored to the estimation of the two-parameter asymmetric input nonlinearity of the VSV actuation system model.

The actuation system considered in this work is manufactured by Rolls-Royce Control Systems (RRCS) in Birmingham, UK. The simplified model and load estimation algorithm developed in this work are obtained and validated using the Trent 1000 Real System Simulator (RSS) implemented in Simulink. The simulations generated by the Trent 1000 RSS are considered to be very accurate and are employed by RRCS as surrogate experimental data for development and certification purposes. It will be shown that the simplified model achieves excellent performance with a low computational load.

\section{Fueldraulic servo valve and actuator}

The fueldraulic actuation system treated in this work comprises a two-stage electro-hydraulic servo valve and an hydraulic actuator. A schematic representation of the system is given in Figure 1. The first stage servo valve, together with a mechanical feedback loop between the first and the second stage servo valves, establishes a proportional relation between the control variable TMC (variable stator vanes Torque Motor Current) and the position of the piston of the second stage servo valve. The second stage servo valve, based on the openings of its orifices, establishes supply flows, from a high-pressure manifold at pressure $\mathrm{Hp}$, and return flows, to a low-pressure manifold at pressure $L p$, to and from the actuator's chambers. These pressures are determined by the engine speed and can be assumed to be constant in a given flight condition (e.g. 'Ground idle', 'Cruise', etc..). In extension, orifices 1 and 4 are open, and orifices 3 and 6 are closed, while, in retraction, orifices 3 and 6 are open and orifices 1 and 4 are closed. Orifices 2 and 5 are always open. In turn, the supply and return flows alter pressures in the headend $\left(P_{H}\right)$ and in the rod-end $\left(P_{R}\right)$ chambers causing movement of the piston. The position of the piston is the measurable output of the system and is denoted by $X_{V S V A}$. In the diagram of Figure 1, note the presence of the cooling flow orifice.

In this section, the equations that represent the system in the Trent 1000 RSS are provided. In a preliminary conference contribution, ${ }^{29}$ a simplified version of the actuation system was considered which omitted the cooling flow. Here, a complete representation of the system is provided, with additional technical explanations, and, crucially, including the cooling flow, which, as it will be shown in the following sections, has a substantial effect on the system's behaviour. It is pointed out that some characteristics of the actuation system are omitted due to confidentiality. However, these characteristics are entirely outside the scope of the analysis and the equations provided constitute a realistic description of the system. In addition, a full set of representative values of the parameters is provided in the section "Simulation results", which would allow the interested reader to simulate the simplified model.

The movement of the piston is described by:

$$
\ddot{x}_{V S V A}=\frac{1}{M}\left(F_{P}+F_{L}\right)
$$

where $M$ is its mass and $F_{P}$ and $F_{L}$ are internal and external loads. The convention adopted is that forces acting on the piston take a positive sign when directed towards extension. The force $F_{P}$ is developed by the internal pressures acting on the piston. It is given by:

$$
F_{P}=A_{H} \cdot P_{H}-A_{R} \cdot P_{R}
$$

where $A_{R}$ and $A_{H}$ are the rod-end and head-end piston's surfaces. Note that $A_{H}>A_{R}$ (see Figure 1). The force $F_{L}$ is an exogenous variable which acts always against the actuator's movement. In addition, according to performance data, $F_{L}$ is stronger in 
retracting mode than in extending mode. This is explained as follows. The external load is interpreted to be the sum of aerodynamic $\left(F_{A}\right)$ and friction $\left(F_{F}\right)$ loads:

$$
F_{L}=F_{A}+F_{F} .
$$

The aerodynamic load $F_{A}$ acts on the stator vanes and is always positive (i.e. supporting extension). The friction $F_{F}$ acts against the actuator movement and is mainly developed in the vanes' pivots and in the transmission from the actuator to the vanes. This is a complex mechanical linkage, containing a large amount of moving joints. In comparison, the friction forces inside the actuator result to be negligible. Hence, the aerodynamic load can be assumed to be always lower than the friction. Finally, in a given flight condition, $F_{A}$ and the modulus of $F_{F}$ are assumed to be constant (see more details at the end of the section). Hence, $F_{L}$ assumes only two possible values: $F_{L}^{E}$ (negative, in extension) or $F_{L}^{R}$ (positive, in retraction).

The pressures $P_{H}$ and $P_{R}$ are described by compressible flow equations:

$$
\begin{aligned}
& \dot{P}_{H}=\frac{B}{V_{H}}\left(Q_{H}+Q_{H d}-Q_{C}\right) \\
& \dot{P}_{R}=\frac{B}{V_{R}}\left(Q_{R}+Q_{R d}+Q_{C}\right)
\end{aligned}
$$

where $Q_{R}$ and $Q_{H}$ are flows through the secondstage servo valve and into or from the rod-end and head-end chambers respectively, $Q_{R d}$ and $Q_{H d}$ are displacement flows caused by the piston's movement, and $Q_{C}$ is the cooling flow. All flows, apart from the cooling flow, take positive sign when incoming (i.e. raising pressures). In extension, $Q_{H}$ is positive (flow through orifices 1 and 2) and $Q_{R}$ is negative (flow through orifices 5 and 4). In retraction, $Q_{H}$ is negative (flow through orifices 2 and 3 ) and $Q_{R}$ is positive (flow through orifices 6 and 5). The cooling flow $Q_{C}$ is considered positive from head-end to rodend, regardless of the piston's motion. In addition, $B$ is bulk modulus, which characterises the fuel's compressibility, and $V_{H}$ and $V_{R}$ are the actuator chambers' volumes. These volumes are function of the piston position, but their variation is assumed to be negligible. This can be done because the servovalve is located on the fan case, while the actuator is on the compressor case. The manifold between these two elements is relatively long, and contains a high volume of fluid, in comparison to the volume of fluid in the chambers. This assumption, which in some cases has been already adopted in the literature see e.g. Thompson et al. ${ }^{11}$, Karpenko and Sepehri ${ }^{13}$, introduces a considerable simplification of the equations.

Flows $Q_{H}$ and $Q_{R}$ are determined by the flownumbers of the respective servo valve orifices. The flownumber is a parameter integrating the physical characteristics of the orifices with those of the fluid into a single coefficient in the flow equations (see e.g. Jalali and $\mathrm{Kroll}^{2}$ where the term 'flow coefficient' is used

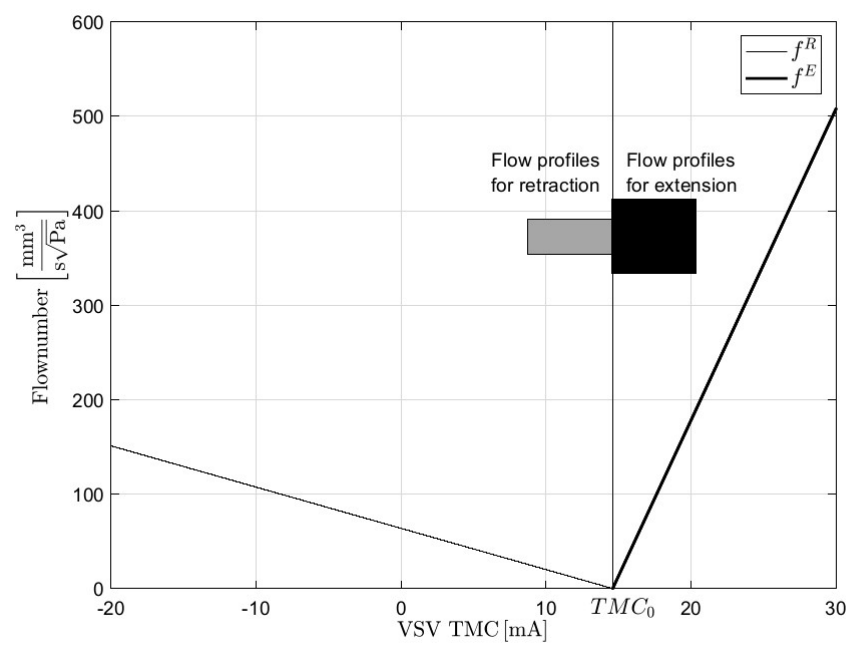

Figure 2. Servo valve flownumbers, with highlighted orifices' profiles for the high-flow extension and low-flow retraction modes

instead). The orifices' flownumbers for $Q_{H}$ and for $Q_{R}$ in both retraction and extension are approximately equal. Hence, the same variables $f^{R}$ and $f^{E}$ are used to denote the flownumbers, in retraction and in extension respectively, for both $Q_{H}$ and $Q_{R}$. The resulting flow equations are:

$$
\begin{aligned}
& Q_{H}= \begin{cases}f^{E} \sqrt{H p-P_{H}}, & \text { in extension, } \\
-f^{R} \sqrt{P_{H}-L p}, & \text { in retraction, }\end{cases} \\
& Q_{R}= \begin{cases}-f^{E} \sqrt{P_{R}-L p}, & \text { in extension, } \\
f^{R} \sqrt{H p-P_{R}}, & \text { in retraction, }\end{cases}
\end{aligned}
$$

where $f^{R}$ and $f^{E}$ are determined by the openings of the servo valve orifices as described in the ensuing equations (11), (12), and Figure 2. The remaining flows are described by:

$$
\begin{aligned}
Q_{H d} & =-A_{H} \cdot \dot{x}_{V S V A}, \\
Q_{R d} & =A_{R} \cdot \dot{x}_{V S V A}, \\
Q_{C} & =f_{C} \operatorname{sgn}\left(P_{H}-P_{R}\right) \sqrt{\left|P_{H}-P_{R}\right|} .
\end{aligned}
$$

Note that the superscripts $\mathrm{E}$ and $\mathrm{R}$ distinguish between extending and retracting modes (as in $f^{E}$ and $f^{R}$ ) while subscripts $\mathrm{R}$ and $\mathrm{H}$ distinguish between the rod-end and the head-end chambers (as in $P_{H}$ and $P_{R}$ ).

The steady-state relations between input current $T M C$ and the flownumbers $f^{E}$ and $f^{R}$ are described by:

$$
\begin{aligned}
& f^{E}= \begin{cases}K^{E}\left(T M C-T M C_{0}\right), & T M C \geq T M C_{0}, \\
0, & T M C<T M C_{0},\end{cases} \\
& f^{R}= \begin{cases}0, & T M C \geq T M C_{0}, \\
K^{R}\left(T M C_{0}-T M C\right), & T M C<T M C_{0},\end{cases}
\end{aligned}
$$


as displayed in Figure 2. The dynamics of the servo valve includes the drive circuit and motor lags and the motion of the piston. This is represented by a damped third order system with a cutoff frequency at around $10 \mathrm{~Hz}$.

The flownumbers profiles depicted in Figure 2 implement the safety requirement that the VSV actuator should slowly retract in case of a power interrupt. This loads the compressor and slows down the engine, thus preventing an engine surge in case of electrical power loss. This behaviour is obtained by means of a positive $T M C_{0}$ value, i.e. the current corresponding to all orifices closed, and by means of asymmetric profiles of the orifices. In particular, the reduced gain of the flownumber profile in the retracting mode allows for a slow retraction in the case that $T M C=0$, i.e. a power interrupt.

In summary, the dynamics of the VSV actuation system in the Trent 1000 RSS is described by a nonlinear dynamical system (equations (1)-(12)) with three states (velocity $\dot{x}_{V S V A}$, head-end pressure $P_{H}$ and rod-end pressure $P_{R}$ ), one input (the control input $T M C$ ) and three exogenous variables (high-pressure $H p$ and low-pressure $L p$, entering in (6) and (7), and the actuator's loading $F_{L}$ in (1)). Note that the nonlinearity of the system is due to the flow equations (6-10). The Electronic Engine Controller (EEC) of RollsRoyce engines maintains accurate estimates of $\mathrm{Hp}$ and $L p$ (which are needed for the control of the fuel distribution). Hence these variables can be assumed to be known with sufficient accuracy. Instead, in the Trent 1000 RSS, the aerodynamic loading $F_{A}$ and the modulus of the friction force $F_{F}$ are calculated as functions of the engine speed on the basis of a nominal relation. Hence, they can also be assumed to be constant in a given flight condition. However, in real operating conditions, the relation with engine speed is subject to variations caused by air properties, engine conditions, and other factors. Hence, in a given flight condition, $F_{A}$ and the modulus of $F_{F}$ can be considered to be constant but uncertain.

\section{Operating points and linearisation}

In this section, in view of the formulation of a simplified model, a linearisation based analysis of the system is developed. In order to find the operating point $\ddot{x}_{V S V}, \dot{P}_{H}$, and $\dot{P}_{R}$ are set to 0 . The derivations are carried out separately for the extending and retracting modes because different flow equations apply. In addition, since the input TMC affects the actuator only through the static relations (11) and (12), in this section, in order to simplify the notation, the flownumber $f^{E}$ (in extending mode), or $f^{R}$ (in retracting mode), are considered to be the actuator's input. The subscript 0 denotes input and state variables at the operating point. In addition, $v_{0}$ denotes $\dot{x}_{V S V A}$ at the operating point. The exogenous variables $H p, L p$ and $F_{L}^{E}$ (in extending mode) or $F_{L}^{R}$ (in retracting mode) are assumed to be constant. Recall that $F_{L}^{E}$ is negative and $F_{L}^{R}$ is positive, i.e. always opposing the actuator's movement.

In the following subsections, the operating points for the extending and retracting modes are obtained and then the linearised dynamics is analysed.

\section{Extending mode}

From equations (4), (5) and (6) - (10) one obtains

$$
\begin{aligned}
& f_{0}^{E} \sqrt{H p-P_{H 0}}-A_{H} v_{0}- \\
& \quad-f_{C} \operatorname{sgn}\left(P_{H 0}-P_{R 0}\right) \sqrt{\left|P_{H 0}-P_{R 0}\right|}=0 \\
& -f_{0}^{E} \sqrt{P_{R 0}-L p}+A_{R} v_{0}+ \\
& \quad+f_{C} \operatorname{sgn}\left(P_{H 0}-P_{R 0}\right) \sqrt{\left|P_{H 0}-P_{R 0}\right|}=0,
\end{aligned}
$$

and from equations (1) - (3) one obtains

$$
A_{H} P_{H 0}-A_{R} P_{R 0}+F_{L}^{E}=0 .
$$

From equations (13) and (14), one obtains that the ratio of the head-end and rod-end flows can be expressed as

$$
\begin{aligned}
& \frac{\sqrt{P_{R 0}-L p}}{\sqrt{H p-P_{H 0}}}= \\
& =\frac{A_{R} v_{0}+f_{C} \operatorname{sgn}\left(P_{H 0}-P_{R 0}\right) \sqrt{\left|P_{H 0}-P_{R 0}\right|}}{A_{H} v_{0}+f_{C} \operatorname{sgn}\left(P_{H 0}-P_{R 0}\right) \sqrt{\left|P_{H 0}-P_{R 0}\right|}} .
\end{aligned}
$$

It is now assumed that if $v_{0}>0$ then the cooling flow becomes negligible with respect to the displacement flow. The assumption is justified by considering the small flownumber of the cooling orifice. Under this approximation, from equation (16) one obtains that

$$
\frac{\sqrt{P_{R 0}-L p}}{\sqrt{H p-P_{H 0}}} \approx \frac{A_{R}}{A_{H}} .
$$

The approximate operating pressures $P_{R 0}^{E}$ and $P_{H 0}^{E}$ are now defined as the solution obtained from equations (15) and (17) (the latter with an equals sign). The expressions of the approximate operating pressures are

$$
\begin{aligned}
& P_{R 0}^{E}=\frac{A_{R}^{2} A_{H} H p+A_{H}^{3} L p+A_{R}^{2} F_{L}^{E}}{A_{H}^{3}+A_{R}^{3}}, \\
& P_{H 0}^{E}=\frac{A_{R}^{3} H p+A_{R} A_{H}^{2} L p-A_{H}^{2} F_{L}^{E}}{A_{H}^{3}+A_{R}^{3}},
\end{aligned}
$$

and the corresponding cooling flow is given by

$$
Q_{C 0}^{E}=f_{C} \operatorname{sgn}\left(P_{H 0}^{E}-P_{R 0}^{E}\right) \sqrt{\left|P_{H 0}^{E}-P_{R 0}^{E}\right|} .
$$

Expressions (18) and (19) are valid provided that $A_{H} H p-A_{R} L p+F_{L}^{E}>0$ which ensures that $H p>$ $P_{H 0}^{E}$ and $P_{R 0}^{E}>L p$. Note that for given $H p$ and $L p$ the inequality provides the maximum loading against which the extending mode can be established.

From the equations above, it can be noticed that, under the approximation introduced, the operating 
pressures $P_{H 0}^{E}, P_{R 0}^{E}$ depend on $H p, L p$ and $F_{L}^{E}$ but are independent from the input $f_{0}^{E}$. Then, for a given flownumber $f_{0}^{E}$, one obtains the operating point velocity $v_{0}$ from equations (13) and (14). However, note that, since $P_{R 0}^{E}$ and $P_{H 0}^{E}$ were based the approximation (17), they would not fulfil (13) and (14) for the same $v_{0}$. Thus, an approximate expression $v_{0}^{E}$ of the operating velocity $v_{0}$ is obtained as the arithmetic mean of the operating velocities obtained from (13) and (14). This is given by:

$$
v_{0}^{E}=\frac{1}{A_{H}} \sqrt{H p-P_{H 0}^{E}}\left(f_{0}^{E}-f_{S}^{E}\right)
$$

where

$$
f_{S}^{E}=\frac{1}{2} \frac{A_{R}+A_{H}}{A_{R}} \frac{Q_{C 0}^{E}}{\sqrt{H p-P_{H 0}^{E}}} .
$$

In (21), $f_{S}^{E}$ represents explicitly the minimum value of $f_{0}^{E}$ corresponding to a positive $v_{0}^{E}$. Note that, since in the derivations a positive velocity is assumed, the expression on the right-hand side of (21) is meaningful only for positive values of $v_{0}^{E}$. Hence, it can be concluded that, for given $H p, L p$ and $F_{L}^{E}$, the extending mode is established for $f_{0}^{E}>\max \left(0, f_{S}^{E}\right)$.

In extending mode, the two cases $P_{H 0}^{E}>P_{R 0}^{E}$ and $P_{H 0}^{E}<P_{R 0}^{E}$ can arise. For given $H_{p}$ and $L_{p}$, the actuator loading $F_{L}^{E}$ discriminates between the two cases. From (18) and (19) one obtains that $P_{H 0}^{E}=P_{R 0}^{E}$ when the actuator loading is equal to

$$
\bar{F}_{L}^{E}=\frac{\left(A_{R}-A_{H}\right)\left(A_{R}^{2} H p+A_{H}^{2} L p\right)}{A_{H}^{2}+A_{R}^{2}} .
$$

Note that $\bar{F}_{L}^{E}$ is negative, since $A_{H}>A_{R}$. Since $F_{L}^{E}$ is also negative, the two cases can arise: $F_{L}^{E}<\bar{F}_{L}^{E}$ and $F_{L}^{E}>\bar{F}_{L}^{E}$. If $F_{L}^{E}<\bar{F}_{L}^{E}$, then $P_{H 0}^{E}>P_{R 0}^{E}, Q_{C 0}^{E}$ is positive (i.e. from head-end to rod-end) and $f_{S}^{E}>0$. If $F_{L}^{E}>\bar{F}_{L}^{E}$, $Q_{C 0}^{E}$ is negative (i.e. from rod-end to head-end) and $f_{S}^{E}<0$. The difference between the two cases is now illustrated. If $F_{L}^{E}<\bar{F}_{L}^{E}$ and $0<f_{0}^{E}<f_{S}^{E}$ then the valve opening is not enough to establish an incoming flow which is bigger than the cooling flow and so $v_{0}^{E}=0$. Hence, if $F_{L}^{E}<\bar{F}_{L}^{E}$, the extending mode is established only for $f_{0}^{E}>f_{S}^{E}$. If $F_{L}^{E}>\bar{F}_{L}^{E}$ then the flow coming into the actuator has direction opposite to that of the cooling flow. In this case any $f_{0}^{E}>0$ is sufficient to establish the extending mode. In particular, a strictly positive $v_{0}^{E}$ is established for $f_{0}^{E}=0^{+}$.

Illustrative examples of the relation between $f_{0}^{E}$ and $v_{0}^{E}$ are shown in Figure 3 (b) for selected flight conditions: 'Ground idle', 'Take-off', 'Cruise' and 'Descent idle'. In each case, the corresponding values of $H p, L p$, and $F_{L}^{E}$ were obtained from the Trent 1000 RSS. In the case denoted 'Cruise outlier' the actuator loading is $20 \%$ lower than the nominal actuator loading. In the other cases the loading takes nominal values. It can be seen from the figure that $f_{S}^{E}>0$, in the four cases corresponding to nominal conditions, while $f_{S}^{E}<0$ in the case 'Cruise outlier'. In addition, note that the slope of the linear part is different for each flight condition.

\section{Retracting mode}

Following a derivation similar to that presented for the extending mode, with the obvious modification in the notation, one obtains the following expressions which define the operating point in the retracting mode:

$$
\begin{aligned}
P_{R 0}^{R} & =\frac{A_{H}^{3} H p+A_{R}^{2} A_{H} L p+A_{R}^{2} F_{L}^{R}}{A_{R}^{3}+A_{H}^{3}}, \\
P_{H 0}^{R} & =\frac{A_{R} A_{H}^{2} H p+A_{R}^{3} L p-A_{H}^{2} F_{L}^{R}}{A_{R}^{3}+A_{H}^{3}}, \\
Q_{C 0}^{R} & =-f_{C} \sqrt{P_{R 0}^{R}-P_{H 0}^{R}} \\
v_{0}^{R} & =-\frac{1}{A_{R}} \sqrt{H p-P_{R 0}^{R}}\left(f_{0}^{R}-f_{S}^{R}\right), \\
f_{S}^{R} & =\frac{1}{2} \frac{A_{H}+A_{R}}{A_{H}} \frac{-Q_{C 0}^{R}}{\sqrt{H p-P_{R 0}^{R}}} .
\end{aligned}
$$

In this case the expressions are valid provided that $A_{R} H p-A_{H} L p-F_{L}^{R}>0$ which ensures that $H p>P_{R 0}^{R}$ and $P_{H 0}^{R}>L p$.

In the retracting mode, in contrast to the extending mode, the actuator's movement can be established only if $P_{H 0}^{R}<P_{R 0}^{R}$. This is because the rod-end area of the piston is smaller than its head-end area (see Figure 1). Therefore, since $F_{L}^{R}$ is positive, the rod-end pressure must be greater than the head-end pressure in order to enable retraction. This fact can be recovered from the equations. Using (24)-(28) it can be shown that $P_{H 0}^{R}=$ $P_{R 0}^{R}$ is obtained when the actuator's load is equal to

$$
\bar{F}_{L}^{R}=\frac{\left(A_{R}-A_{H}\right)\left(A_{H}^{2} H p+A_{R}^{2} L p\right)}{A_{H}^{2}+A_{R}^{2}} .
$$

Note that $\bar{F}_{L}^{E}$ is negative, since $A_{H}>A_{R}$. However $F_{L}^{R}$ is positive. Hence only the case $F_{L}^{R}>\bar{F}_{L}^{R}$ is possible which, in turn, corresponds to having $f_{S}^{R}>0$. Hence, $v_{0}^{R}=0$ for $0<f_{0}^{R}<f_{S}^{R}$, and the retracting mode is established for $f_{0}^{R}>f_{S}^{R}$. The expression of $\bar{F}_{L}^{R}$ in (29), which appears to be of theoretical interest only, nevertheless will be used to shorten the expressions used to define the simplified model in the next section. The relation between $f_{0}^{R}$ and $v_{0}^{R}$ is illustrated in Figure 3 (a) for the same flight conditions considered in Figure 3 (b).

\section{Linearised models}

Given $H p, L p$ and $F_{L}^{E / R}$, linearised models around operating points $\left[P_{H 0}^{E / R}, P_{R 0}^{E / R}, f_{0}^{E / R}\right]$ are obtained by linearising the flow equations (6), (7) and (10). The linearised system has a pair of high-frequency lightlydamped poles $\left(\approx 10^{3} \mathrm{~Hz}\right)$. In addition, it can be seen that the choice of the operating points has very little effect on the linearised dynamics since it affects only the amplitude of the high-frequency resonance 


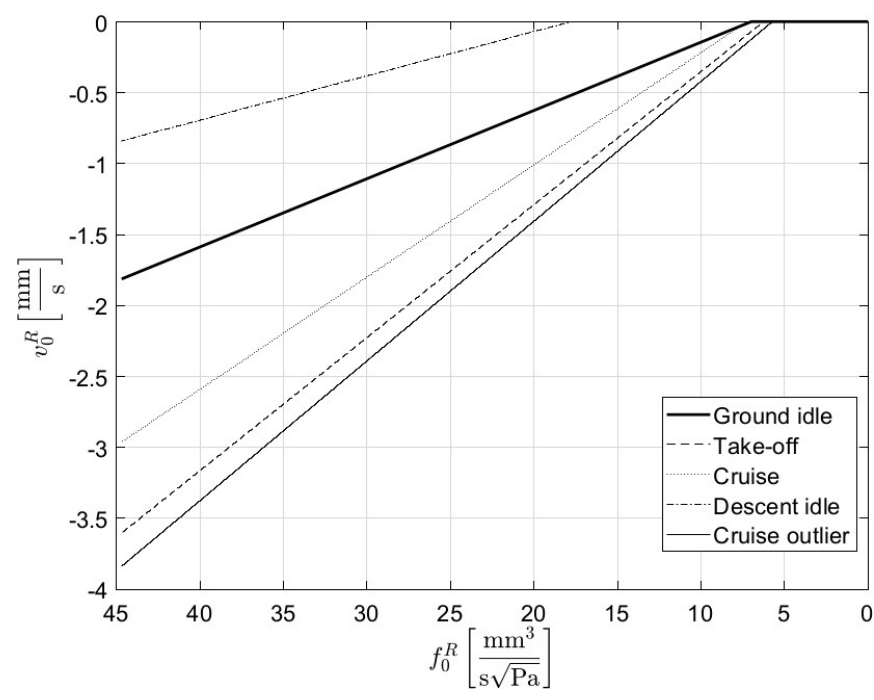

(a) retracting mode

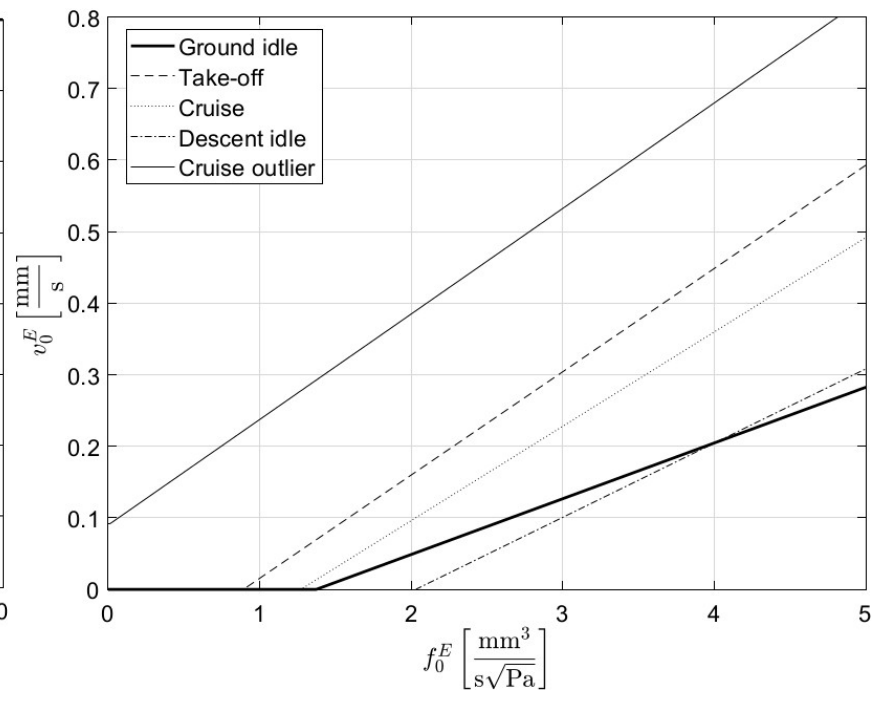

(b) extending mode

Figure 3. Relation between flownumber and velocity in extending and retracting modes for different flight conditions and actuator loadings. (The profiles shown are for two actuators in parallel in accordance with the simulation cases presented in the last section.)

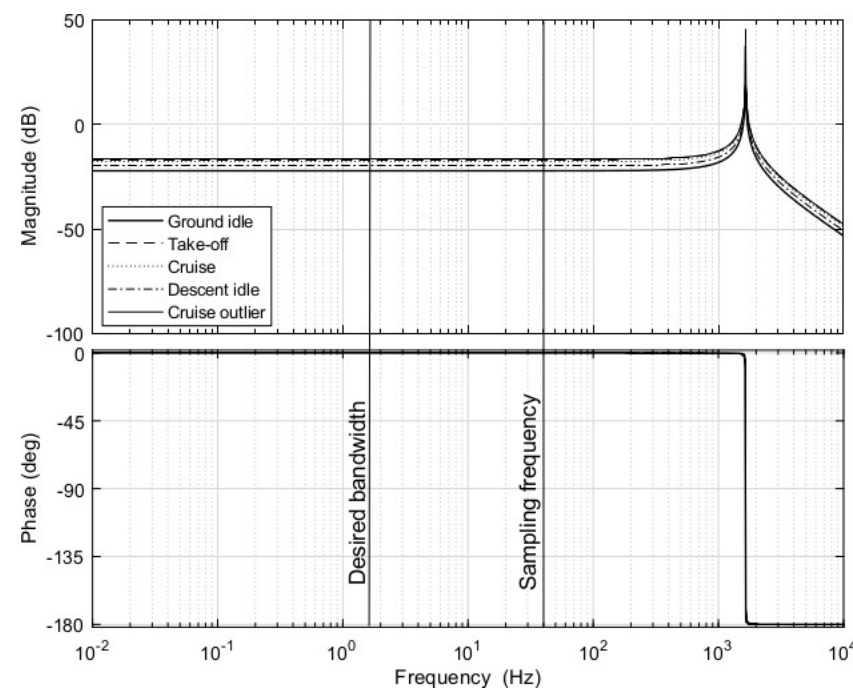

Figure 4. Bode plots of the linearised dynamics between flownumber and velocity in extending mode for the same flight conditions considered in Figure 3(b). The vertical lines indicate the bandwidth of the VSV actuation control system and the sampling frequency.

peak. This is illustrated in Figure 4 where the Bode plots of the linearised dynamics from $f^{E}$ to $\dot{x}_{V S V A}$ corresponding to the flight conditions considered in Figure $3(\mathrm{~b})$. In each flight condition, changing $f_{0}^{E}$ does not cause noticeable changes in the Bode plots. In each Bode plot, the low-frequency gain corresponds to the slope of the linear part of the corresponding graph in Figure 3 (b).

High-frequency lightly-damped poles are a common occurrence in hydraulic systems, as shown in e.g. Fales ${ }^{4}$, Thompson et al. ${ }^{11}$, Karpenko and Sepehri ${ }^{14}$ and Rubagotti et al. ${ }^{21}$. Here, the bandwidth of the VSVA control system is $10 \mathrm{rad} \mathrm{s}^{-1} / 1.6 \mathrm{~Hz}$ and the available sampling frequency is $40 \mathrm{~Hz}$. Hence, the high frequency resonance peak is well beyond the control bandwidth (see Figure 4).

\section{Simplified model}

In this section, a simplified control-oriented model is presented, which approximates the dynamics between the control input $\triangle T M C=T M C-T M C_{0}$ and the actuator's position $x_{V S V A}$. The rationale behind the simplified model is that, following the linearisationbased analysis at different operating points performed in the previous section, the high-frequency dynamics can be neglected in order to represent the behaviour of the actuator in the frequency range of interest. In particular, over the frequency range up to the sampling frequency $40 \mathrm{~Hz}$, it can be assumed that $\triangle T M C$ is linked to $\dot{x}_{V S V A}$ by a delay plus a static nonlinearity.

In order to simplify the notation, the 'signed flownumber' $f$ is introduced to represent both extending and retracting flownumbers with one single variable. In addition, the dynamics of the servo valve inside the bandwidth is well represented by a pure delay $T_{d}=0.025 \mathrm{~s}$. Hence, from (11) and (12) one obtains the static relations

$$
f= \begin{cases}K^{E} \Delta T M C_{d}, & \Delta T M C_{d} \geq 0, \\ K^{R} \Delta T M C_{d}, & \Delta T M C_{d}<0 .\end{cases}
$$

where $\triangle T M C_{d}$ is the delayed $\triangle T M C$. Then, one can link $f$ to $\dot{x}_{V S V A}$ through the non-linear static relation

$\dot{x}_{V S V A}=\left\{\begin{array}{l}N^{E}\left(f-f_{S}^{E}\right), \quad f \geq \max \left(0, f_{S}^{E}\right), \\ 0, \quad-f_{S}^{R}<f<\max \left(0, f_{S}^{E}\right), \\ N^{R}\left(f+f_{S}^{R}\right), \quad f \leq-f_{S}^{R},\end{array}\right.$

where $N^{E}$ and $f_{S}^{E}$ are functions of $F_{L}^{E}, H p, L p$ and $N^{R}$ and $f_{S}^{R}$ are functions of $F_{L}^{R}, H p, L p$ as follows. 


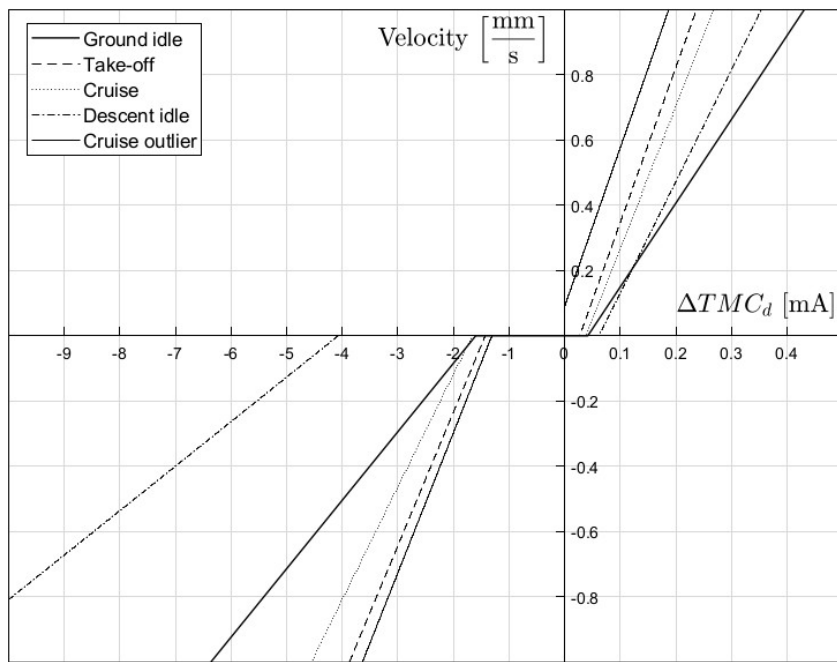

Figure 5. Input nonlinearity for selected flight conditions. Note the different scalings for positive and negative values of $\triangle T M C_{d}$ (compare with Figures 2 and 3 ).

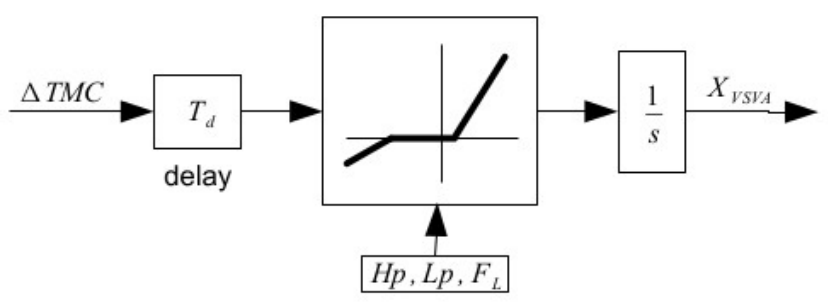

Figure 6. Simplified model of the actuation system

Substituting (18) in (21) and (24) in (27), one obtains

$$
\begin{aligned}
N^{E} & =\sqrt{\frac{A_{H} H p-A_{R} L p+F_{L}^{E}}{A_{H}^{3}+A_{R}^{3}}}, \\
N^{R} & =\sqrt{\frac{A_{R} H p-A_{H} L p-F_{L}^{R}}{A_{H}^{3}+A_{R}^{3}}} .
\end{aligned}
$$

Similarly, using (22) and (28) and taking into account that in extension the cooling flow can be positive or negative, one obtains

$$
\begin{aligned}
f_{S}^{E}= & \operatorname{sgn}\left(\bar{F}_{L}^{E}-F_{L}^{E}\right) \times \\
& \times \frac{f_{C}}{2} \frac{A_{R}+A_{H}}{A_{R} A_{H}} \sqrt{\frac{\left(A_{H}^{2}+A_{R}^{2}\right)\left|\bar{F}_{L}^{E}-F_{L}^{E}\right|}{A_{H} H p-A_{R} L p+F_{L}^{E}}}, \\
f_{S}^{R}= & \frac{f_{C}}{2} \frac{A_{R}+A_{H}}{A_{R} A_{H}} \sqrt{\frac{\left(A_{H}^{2}+A_{R}^{2}\right)\left(F_{L}^{E}-\bar{F}_{L}^{E}\right)}{A_{R} H p-A_{H} L p-F_{L}^{R}}} .
\end{aligned}
$$

Instances of the static nonlinearity obtained by combining (30) and (31) are shown in Figure 5.

In conclusion, the simplified model of the VSV actuator linking $\triangle T M C$ to $x_{V S V A}$ is composed of a pure delay, an input static nonlinearity, and a linear integrator plant (see Figure 6). The coefficients of the static nonlinearity include some physical parameters of the actuator and the exogenous variables $H p, L p$ and $F_{L}^{E / R}$. Some other parameters of the full nonlinear model become irrelevant in the simplified model, these are: the head-end and rod-end volumes of the actuator $\left(V_{H / R}\right)$ and the bulk modulus $(B)$. However, it is remarked that $V_{H / R}$ and $B$ were needed to perform the linearisation analysis of the previous section which led us to the simplified model.

\section{Estimation of the actuator's loading}

In a given flight condition, as discussed in Section 2, $F_{L}^{E}$ and $F_{L}^{R}$ can be considered to be uncertain constants, while accurate values of $H p$ and $L p$ are available in the EEC. Here, It is shown that $F_{L}^{E}$ and $F_{L}^{R}$ can be estimated by a simple extension of the method presented in $\mathrm{Bai}^{28}$. In this section, in order to simplify the notation, the symbols $u=\Delta T M C_{d}, v=\dot{x}_{V S V A}$, and $y=x_{V S V A}$ are used.

To start with, the parametrisation of the 'deadzone' and 'preload' nonlinearities of $\mathrm{Bai}^{28}$ is adopted to express the static nonlinearity in the model. In order to do this, the following coefficients are defined

$$
\begin{aligned}
M^{E} & =N^{E} K^{E}, & M^{R} & =N^{R} K^{R}, \\
\bar{u}^{E} & =f_{s}^{E} / K^{E}, & \bar{u}^{R} & =-f_{s}^{R} / K^{R} .
\end{aligned}
$$

Then, by combining (30) and (31) one obtains

$v= \begin{cases}\frac{M^{E}}{2}\left[1+\operatorname{sgn}\left(u-\bar{u}^{E}\right)\right]\left(u-\bar{u}^{E}\right), & u \geq 0 \\ \frac{M^{R}}{2}\left[1-\operatorname{sgn}\left(u-\bar{u}^{R}\right)\right]\left(u-\bar{u}^{R}\right), & u<0 .\end{cases}$

In the above expressions $M^{E}$ and $\bar{u}^{E}$ are functions of $F_{L}^{E}$ and $M^{R}$ and $\bar{u}^{R}$ are functions of $F_{L}^{R}$. Note that $M^{E}$ and $M^{R}$ are always positive. The coefficient $\bar{u}^{R}$ is always negative (deadzone in retraction), while $\bar{u}^{E}$ can be positive (deadzone in extension) or negative (preload in extension).

Given a set of input $(u)$ and output $(y)$ data one can use (38) to compute an estimate of the actuator's velocity $v\left(u, F_{L}^{E}, F_{L}^{R}\right)$ as a function of $F_{L}^{E}$ and $F_{L}^{R}$. Then, estimates $\hat{F}_{L}^{E}$ and $\hat{F}_{L}^{R}$ can be obtained by direct minimisation of a least-squares criterion. In general the problem is not convex but is two dimensional and, thus, is not computationally intensive. In addition, as pointed out in $\mathrm{Bai}^{28}$, the estimate is consistent and has good properties when the data are affected by noise. In particular, if the data are affected by i.i.d. zeromean Gaussian noise then $\hat{F}_{L}^{E}$ and $\hat{F}_{L}^{R}$ are a maximum likelihood estimator. Here, this property is particularly well suited since the noise affecting the data is lowintensity electrical noise which is well described by the i.i.d. Gaussian assumption (see illustrative results in the following section).

\section{Simulation results}

In this section, the simplified VSVA model is assessed in a comparison with the Trent 1000 RSS. The Trent 1000 RSS is a large Simulink model which includes: Pumping unit, Hydro-Mechanical Unit (HMU), Electronic Engine Control (EEC), and Engine. 


\begin{tabular}{ll}
\hline$K^{R}=4.36 \mathrm{~mm}^{3} \mathrm{~s}^{-1} \mathrm{~Pa}^{-1 / 2} \mathrm{~mA}^{-1}$ & $M=0.90 \mathrm{~kg}$ \\
$K^{E}=32.97 \mathrm{~mm}^{3} \mathrm{~s}^{-1} \mathrm{~Pa}^{-1 / 2} \mathrm{~mA}^{-1}$ & $H p=2061 \mathrm{kPa}$ \\
$A_{H}=5748 \mathrm{~mm}^{2}$ & $L p=358 \mathrm{kPa}$ \\
$A_{R}=4940 \mathrm{~mm}^{2}$ & $F_{L}^{E}=-1664 \mathrm{~N}$ \\
$f_{C}=0.582 \mathrm{~mm}^{3} \mathrm{~s}^{-1} \mathrm{~Pa}^{-1 / 2}$ & $F_{L}^{R}=4430 \mathrm{~N}$ \\
\hline
\end{tabular}

Table 1. Representative values of parameters in 'ground idle'.

In the RSS, the VSVA component includes the servo valve and two actuators in parallel. In the RSS VSVA component, the actuation system is implemented by the full non-linear equations (1)-(12). In Table 1 numerical values of the parameters of the RSS VSVA component, which are representative of a 'ground idle' state, are disclosed. Note that Table 1 includes all parameters which appear in the simplified model. For confidentiality, the parameters' values in the other fight conditions are not disclosed. In order to generate the simulations presented in this section, RSS VSVA component was isolated from the rest of the RSS. Hence $H p, L p, F_{A}$ and $F_{L}^{E / R}$ were set at constant values corresponding to the given flight condition. In order to generate the closed-loop simulations, the existing PI controller from the EEC module was included. The simulations of the RSS VSVA component were generated using the Simulink Runge-Kutta solver with a $10^{-6}$ s fixed-step size compiled in rapid accelerator mode. The reason for the choice of such a short step size is that the RSS VSVA component includes stiff differential equations (see the high-frequency resonance peak shown in Figure 3). In order to generate a 10s response, the solver required approximately two minutes of computational time. In contrast, the simplified model was simulated using a variable-step solver requiring a comparatively negligible time.

In Figures 7 and 8 the position and velocity responses generated by the RSS VSVA component are compared with the responses generated by the simplified model. It can be seen that the responses provided by the simplified model mostly overlap those generated by the RSS. Hence, the simplified model provides computationally light yet accurate simulations. For completeness, the mean squared error (mse) between the two responses, computed with sampling time $0.025 \mathrm{~s}$, is reported for each plot. For the position responses of Figure 7 the values are mse $=3.66 \times 10^{-3}$ and $\mathrm{mse}=1.77 \times 10^{-5}$ for the open and closed-loop responses respectively. For the velocity responses of Figure 8 the values are mse $=1.388 \times 10^{-3}$ and mse $=$ $9.51 \times 10^{-3}$ for the open and closed-loop responses respectively. Note that the mse of the closed-loop position responses is much lower than the mse of the open-loop position responses. This is expected since position is the controlled variable in the closed-loop system. In all cases, the mse values, which are very low in comparison with the excursion of the responses, confirm the accuracy of the simplified model. It can also be seen that the velocity responses generated by the RSS VSVA component are characterised by bursts of high-frequency oscillations at the transition points (see magnified inset in Figure 8). These oscillations match the frequency of the resonance peak seen in Figure 4 . The oscillations are smoothed by integration and do not appear in the position responses. It can be seen, by comparing the velocity responses, that the velocity generated by the simplified model at the transition points follows a smoothed version of the velocity response generated by the RSS.

In Figure 9, the evolution of the TMC inputs is displayed. In the open-loop simulations, the inputs to the RSS and to the simplified model were the same. In the closed-loop simulations, the TMC inputs were generated by two independent closed-loop systems fed by the same reference signal. Note that the TMC inputs are still almost overlapping. In Figure 9 the value of $T M C_{0}$ and the limits of the input deadzone of the simplified model are also displayed. Note that the deadzone is smaller on the positive side (compare with Figure 5). Comparing Figure 9 with Figure 8, it can be seen that the time intervals in which TMC is inside the dead-zone of the simplified model coincide with time intervals in which the velocity is null. This is more evident in the open-loop simulations, in which the input TMC is inside the deadzone for longer intervals.

In Figure 10, the evolution of $P_{H}$ and $P_{R}$ in the simulation generated by the RSS is displayed. In the figure, the operating pressures $P_{H 0}^{E / R}$ and $P_{R 0}^{E / R}$ are also displayed and are plotted only over the time intervals in which the actuators are moving. From the figures it can be seen that the pressure $P_{H}$ and $P_{R}$ converge very rapidly to the operating values whenever the actuator is moving independently of the attained velocity. In this respect, recall that the independence of operating pressure from the velocity was a key point in the formulation of the simplified model. In addition, note that $P_{H}$ and $P_{R}$ are subject to the same bursts of oscillations seen in the velocity plots. However, in the case of pressures, the amplitudes of the oscillations are relatively too small to be seen in the plots.

To conclude, the results of the estimation of the actuator's loads are illustrated. The estimates were obtained using sampled open-loop and closedloop data generated by the RSS and acquired with sampling time $0.025 \mathrm{~s}$. The open-loop and closed-loop inputs were those used to generate the simulations in Figures 7-10. The acquisition of data was simulated by including the transducer component which includes a realistic source of low-intensity electrical noise. The velocity of the actuators was then reconstructed in discrete-time using a backward difference discretisation scheme. ${ }^{30}$ The optimisation of the least-squares estimation criterion was performed by a simple grid search. The estimates, and relative errors with respect to the true values in Table 1, were $\hat{F}_{L}^{E}=-1721 \mathrm{~N}(3.5 \%)$ and $\hat{F}_{L}^{R}=4423 \mathrm{~N}(0.2 \%)$ obtained from the open-loop data, and $\hat{F}_{L}^{E}=-1664 \mathrm{~N}(0 \%)$ and $\hat{F}_{L}^{R}=4430 \mathrm{~N}(0 \%)$ obtained from the closed-loop data. Note that the relative errors range from negligible to $3.5 \%$. Thus, the estimated loads can be considered to be very accurate. The contour plots of the least- 

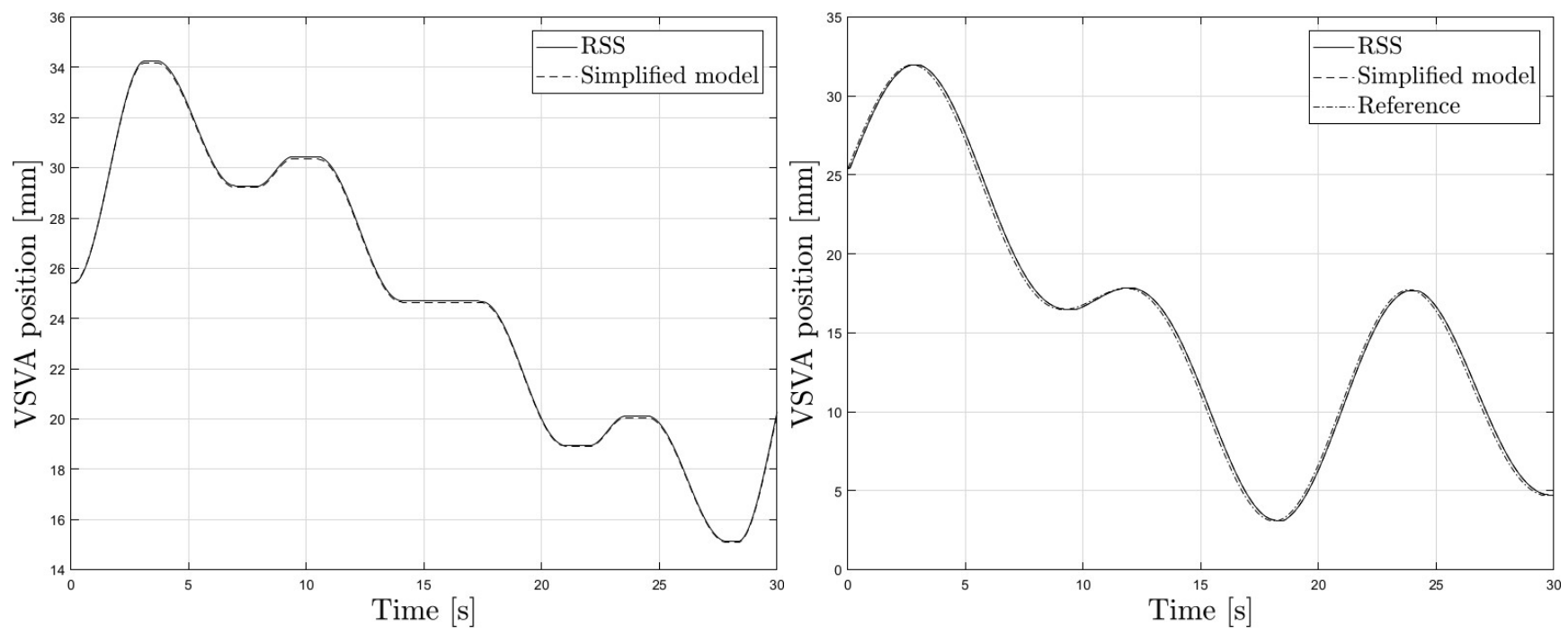

Figure 7. Position responses in open-loop (left) and in closed-loop (right).
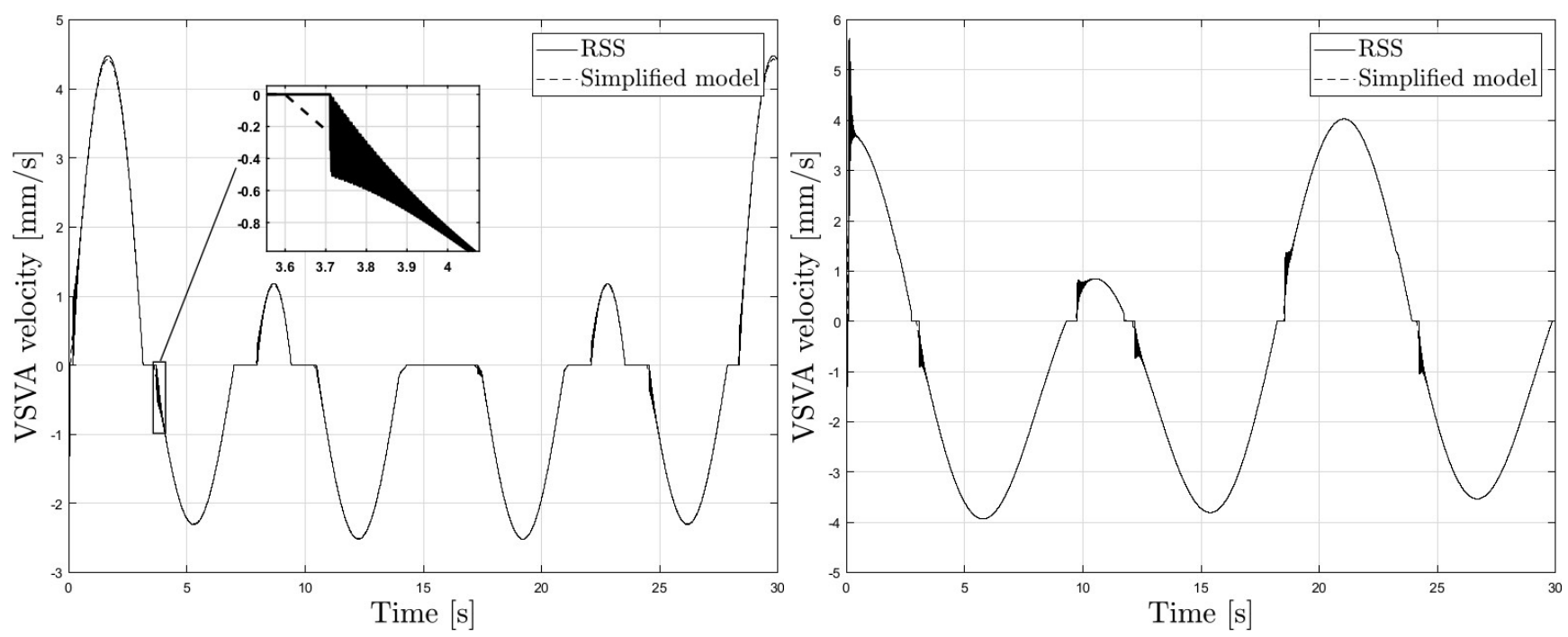

Figure 8. Velocity responses in open-loop (left) and in closed-loop (right).
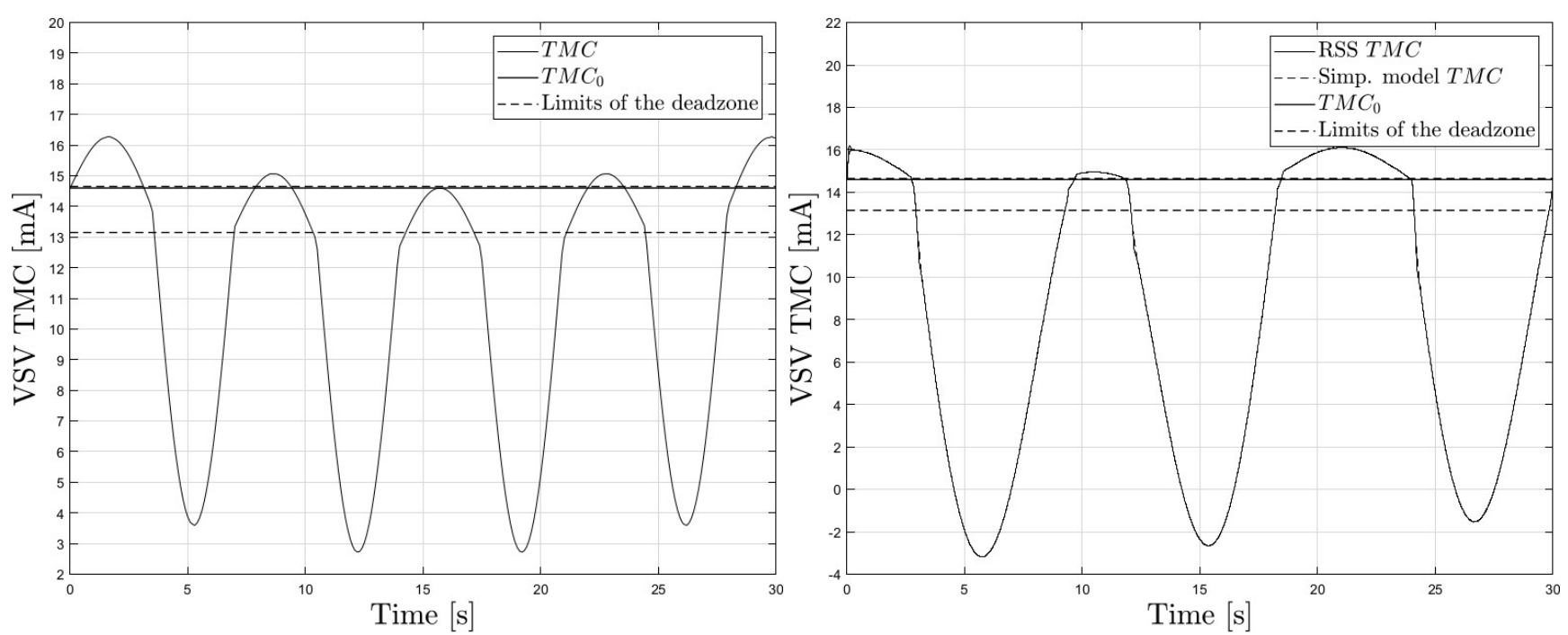

Figure 9. TMC inputs in open-loop (left) and in closed-loop (right). 

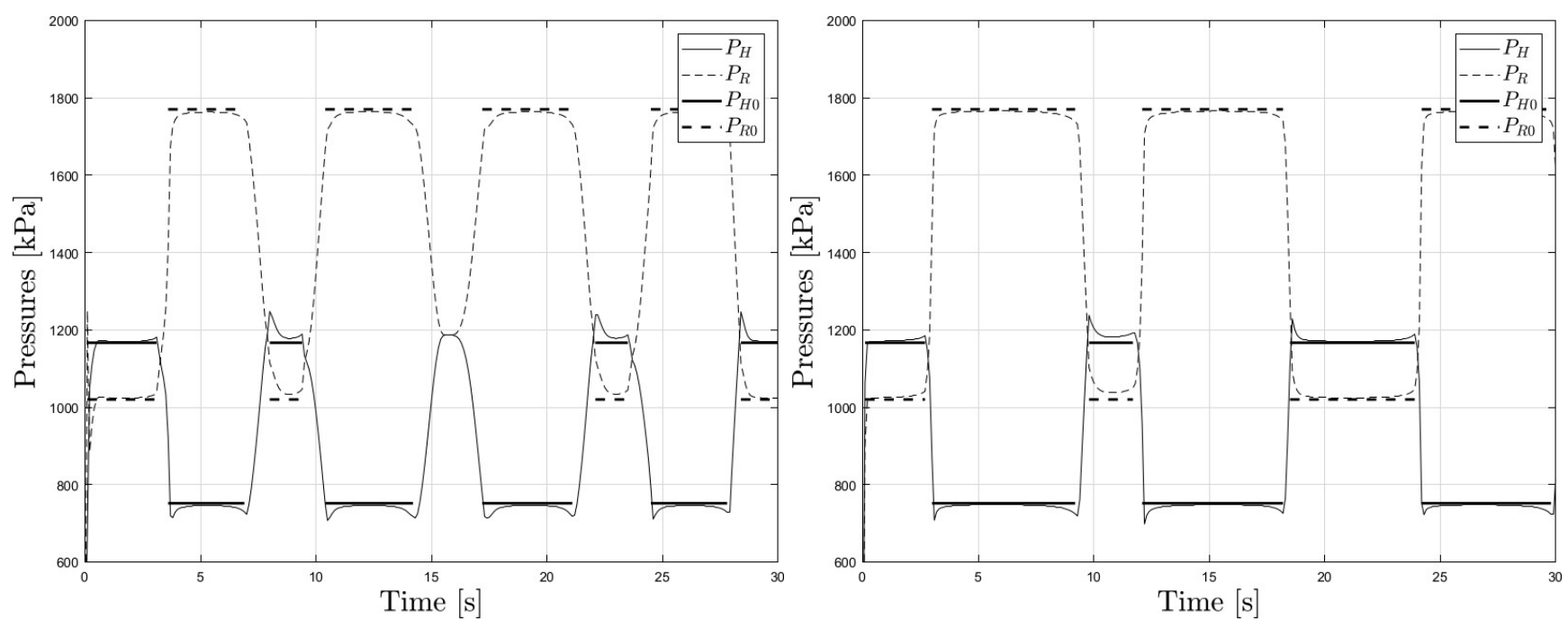

Figure 10. Pressure responses in open-loop (left) and in closed-loop (right).
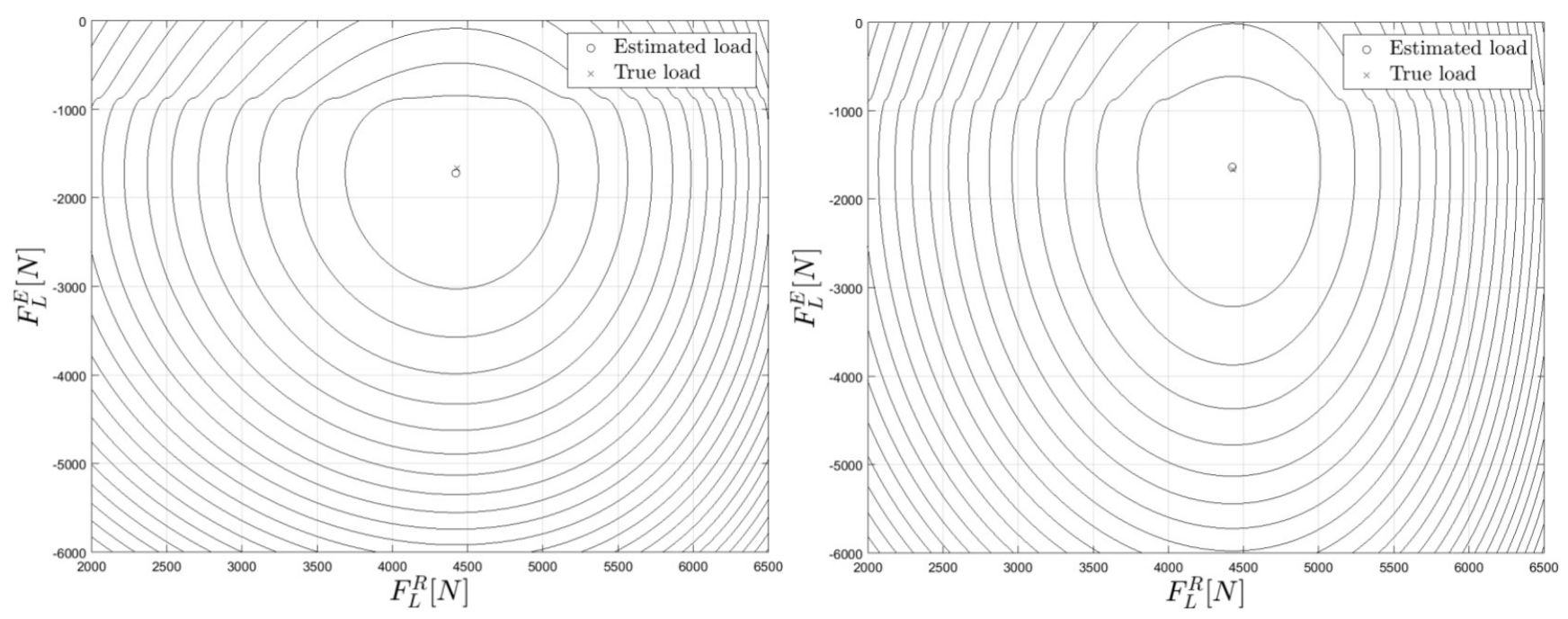

Figure 11. Estimation criterion using open-loop data (left) and using closed-loop data (right).

-squares criteria in both cases are shown in Figure 11. Note how the cost functions are such that the optimum is easily determined. The estimation of the actuator's loads was repeated for all flight conditions considered in Figure 3, which correspond to significantly different shapes of the input nonlinearity in the simplified model, leading to similar results in each case.

\section{Conclusions}

In this paper, a simplified model of the compressor variable stator vanes (VSVA) actuation system of the Rolls-Royce Trent 1000 engine was presented. The actuator is characterised by the presence of a cooling flow orifice which significantly affects its behaviour. It has been found that the behaviour of the actuator is accurately represented by a static input nonlinearity followed by a linear system. The accuracy of the simplified model was verified using the RollsRoyce Trent 1000 Real System Simulator. The VSVA simplified model has enabled us to develop a fast algorithm for computing a snapshot estimate of the actuator's external load in a given flight condition. The external load is by far the largest uncertainty affecting the actuation system. In our current work we are developing a continuous estimator suitable for changing flight conditions with a focus on fault detection $^{31-34}$.

\section{Acknowledgement}

The authors gratefully acknowledge the technical input of Martin Kirkman (Rolls-Royce Control Systems). This work was supported by Rolls-Royce Control Systems, Birmingham, UK.

\section{References}

1. Herbert E Merritt. Hydraulic control systems. John Wiley \& Sons, 1967.

2. Mohieddine Jalali and Andreas Kroll. Hydraulic servosystems. Modeling, Identification and Control, Springer, 2004. 
3. Bora Eryilmaz and Bruce H Wilson. Unified modeling and analysis of a proportional valve. Journal of the Franklin Institute, 343(1):48-68, 2006.

4. Roger Fales. Stability and performance analysis of a metering poppet valve. International Journal of Fluid Power, 7(2):11-17, 2006.

5. Davide Cristofori and Andrea Vacca. Modeling hydraulic actuator mechanical dynamics from pressure measured at control valve ports. Proceedings of the Institution of Mechanical Engineers, Part I: Journal of Systems and Control Engineering, 229(6):541-558, 2015.

6. Amin Maghareh, Christian E Silva, and Shirley J Dyke. Parametric model of servo-hydraulic actuator coupled with a nonlinear system: Experimental validation. Mechanical Systems and Signal Processing, 104:663-672, 2018.

7. GP Liu and S Daley. Optimal-tuning nonlinear pid control of hydraulic systems. Control Engineering Practice, 8(9):1045-1053, 2000.

8. Ill-yeong Lee, Dong-hun Oh, Sang-won Ji, and So-nam Yun. Control of an overlap-type proportional directional control valve using input shaping filter. Mechatronics, 29: 87-95, 2015.

9. Yi Ye, Chen-Bo Yin, Yue Gong, and Jun-jing Zhou. Position control of nonlinear hydraulic system using an improved pso based pid controller. Mechanical Systems and Signal Processing, 83:241-259, 2017.

10. Frans Wijnheijmer, Gerrit Naus, Wil Post, Maarten Steinbuch, and Piet Teerhuis. Modelling and lpv control of an electro-hydraulic servo system. In IEEE International Conference on Control Applications, Munich, Germany, 2006.

11. David F Thompson, John S Pruyn, and Amit Shukla. Feedback design for robust tracking and robust stiffness in flight control actuators using a modified QFT technique. International Journal of Control, 72(16):14801497, 1999.

12. M Karpenko and N Sepehri. Robust position control of an electrohydraulic actuator with a faulty actuator piston seal. ASME Journal of Dynamic Systems, Measurement, and Control, 125(3):413-423, 2003.

13. Mark Karpenko and Nariman Sepehri. Fault-tolerant control of a servohydraulic positioning system with crossport leakage. IEEE Transactions on Control Systems Technology, 13(1):155-161, 2005.

14. Mark Karpenko and Nariman Sepehri. On quantitative feedback design for robust position control of hydraulic actuators. Control Engineering Practice, 18(3):289-299, 2010.

15. Vladimir Milić, Željko Šitum, and Mario Essert. Robust $\mathrm{h}$ position control synthesis of an electro-hydraulic servo system. IS A transactions, 49(4):535-542, 2010.

16. Lisandro J Puglisi, Roque J Saltaren, Cecilia Garcia, and Ilka A Banfield. Robustness analysis of a pi controller for a hydraulic actuator. Control Engineering Practice, 43: 94-108, 2015.

17. G. Ren, M. Esfandiari, J. Song, and N. Sepehri. Position control of an electrohydrostatic actuator with tolerance to internal leakage. IEEE Transactions on Control Systems Technology, 24(6):2224-2232, 2016.
18. J. E. Bobrow and K. Lum. Adaptive, high bandwidth control of a hydraulic actuator. ASME Journal of Dynamic Systems, Measurement, and Control, 118(4):714, 1996.

19. Mete Kalyoncu and Mustafa Haydim. Mathematical modelling and fuzzy logic based position control of an electrohydraulic servosystem with internal leakage. Mechatronics, 19(6):847-858, 2009.

20. Amit Mohanty and Bin Yao. Integrated direct/indirect adaptive robust control of hydraulic manipulators with valve deadband. IEEE/ASME Transactions on Mechatronics, 16(4):707-715, 2011.

21. Matteo Rubagotti, Marco Carminati, Giampiero Clemente, Riccardo Grassetti, and Antonella Ferrara. Modeling and control of an airbrake electro-hydraulic smart actuator. Asian Journal of Control, 14(5):1159-1170, 2012.

22. Kai Guo, Jianhua Wei, Jinhui Fang, Ruilin Feng, and Xiaochen Wang. Position tracking control of electrohydraulic single-rod actuator based on an extended disturbance observer. Mechatronics, 27:47-56, 2015.

23. Hai-Peng Ren and Pei-Fen Gong. Adaptive control of hydraulic position servo system using output feedback. Proceedings of the Institution of Mechanical Engineers, Part I: Journal of Systems and Control Engineering, 231(7):527-540, 2017.

24. Noah D Manring, Laheeb Muhi, Roger C Fales, Viral S Mehta, Jeff Kuehn, and Jeremy Peterson. Using feedback linearization to improve the tracking performance of a linear hydraulic-actuator. Journal of Dynamic Systems, Measurement, and Control, 140(1), 2018.

25. Yong Li and Qingfeng Wang. Adaptive neural finitetime trajectory tracking control of hydraulic excavators. Proceedings of the Institution of Mechanical Engineers, Part I: Journal of Systems and Control Engineering, 2018.

26. Zhikai Yao, Yongping Yu, and Jianyong Yao. Artificial neural network-based internal leakage fault detection for hydraulic actuators: An experimental investigation. Proceedings of the Institution of Mechanical Engineers, Part I: Journal of Systems and Control Engineering, 232(4):369-382, 2018.

27. Fouad Giri and Er-Wei Bai, editors. Block-oriented Nonlinear System Identification. Springer London, 2010.

28. Er-Wei Bai. Identification of linear systems with hard input nonlinearities of known structure. Automatica, 38 (5):853-860, 2002.

29. Tomas Puller and Andrea Lecchini-Visintini. Modelling for control of a jet engine compressor variable stator vanes hydraulic actuator. In 2016 European Control Conference (ECC), 2016.

30. Karl Johan Astrom and Bjorn Wittenmark. ComputerControlled Systems. Dover Publications Inc., 2011.

31. Murat Arcak and Petar Kokotović. Nonlinear observers: a circle criterion design and robustness analysis. Automatica, 37(12):1923-1930, 2001.

32. J. Voros. Recursive identification of hammerstein systems with discontinuous nonlinearities containing dead-zones. IEEE Transactions on Automatic Control, 48 (12):2203-2206, 2003.

33. W.-H. Chen. Disturbance observer based control for nonlinear systems. IEEE/ASME Transactions on Mechatronics, 9(4):706-710, 2004. 
34. Rishi Relan and Johan Schoukens. Recursive discretetime models for continuous-time systems under bandlimited assumptions. IEEE Transactions on Instrumentation and Measurement, 65(3):713-723, 2016. 